\title{
Optimization of Plasmonic Structure Integrated Single-photon Detector Designs to Enhance Absorptance
}

\author{
Mária Csete $^{1, *}$, Gábor Szekeres ${ }^{1}$, Balázs Bánhelyi ${ }^{2}$, András Szenes ${ }^{1}$, Tibor Csendes², Gábor Szabó ${ }^{1}$ \\ ${ }^{I}$ Department of Optics and Quantum Electronics, University of Szeged, H-6720 Szeged, Dóm tér 9, Hungary \\ ${ }^{2}$ Department of Computational Optimization, University of Szeged, H-6720 Szeged, Árpád tér 2, Hungary
}

\begin{abstract}
Plasmonic structure integrated superconducting nanowire single-photon detector (SNSPD) configurations were optimized for $1550 \mathrm{~nm}$ p-polarized light illumination to maximize absorptance. Orientation dependent $\mathrm{NbN}$ absorptance, spectral sensitivity and dispersion characteristics were investigated to find optimal configurations.

OCIS codes: 23.5160 Photodetectors, 240.6680 Surface plasmons, 220.4830 System design
\end{abstract}

\section{Introduction}

Superconducting nanowire single photon detectors (SNSPD) are devices to detect infrared light with photon resolution and high speed. Plasmonic structures integrated into these detector's are capable of enhancing absorptance along with the reduction of kinetic inductance [1, 2].

\section{Methods}

The optical response of SNSPDs consisting of superconducting NbN patterns with user specified parameters was inspected for $1550 \mathrm{~nm}$ polarized infrared light illumination. Namely, $100 \mathrm{~nm}$ wide and $4 \mathrm{~nm}$ thick niobium-nitride $(\mathrm{NbN})$ nanowire array with $792 \mathrm{~nm}$ periodicity commensurate with $3 / 4 * \lambda_{\mathrm{SPP}, 1500 \mathrm{~nm}}$ three-quarter wavelength of SPPs propagating on silica-gold interface was the original pattern.

The integrated plasmonic structure was array of $220 \mathrm{~nm}$ high nano-cavities closed by gold reflector and filled with HSQ in NCAI-SNSPD [1], while two different arrays of $220 \mathrm{~nm}$ long and $100 \mathrm{~nm}$ wide gold segments (deflectors) were embedded into the silica substrate with $792 \mathrm{~nm}$ periodicity in NCDAI- and NCDDAI-SNSPD (Figure 1aa-ac). The deflector's geometric properties were designed according to the literature about different types of plasmonic mirrors [2, 3].

The geometric properties of three detector designs were partially and completely optimized, namely, first the optimal height of nano-cavities and deflectors were determined (Figure 1ba-bc), than all geometrical parameters except the $\mathrm{NbN}$ stripes thickness was varied (Figure 1ca-cc).

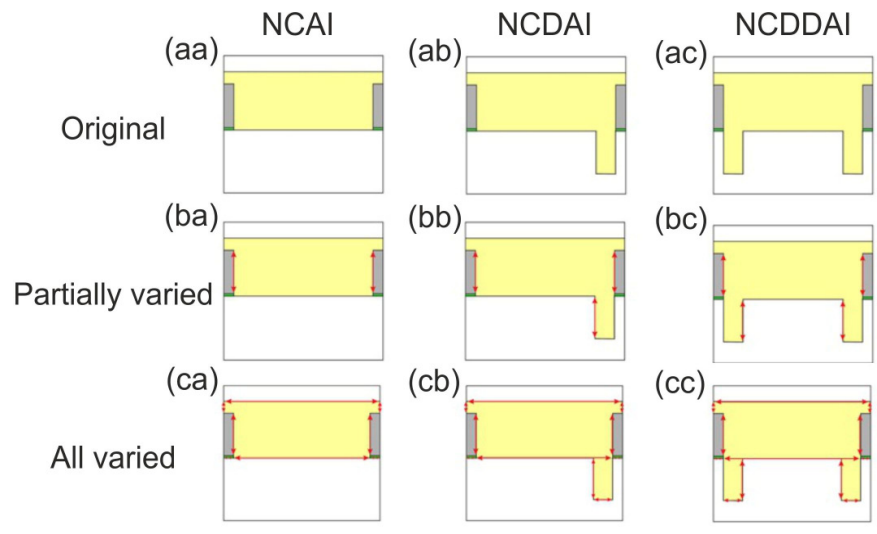

Figure 1. The inspected plasmonic structure integrated single photon-detector designs: NCAI-, NCDAI- and NCDDAI-SNSPD

In order to determine the optimal orientation of three different plasmonic structure integrated SNSPDs, the polar and azimuthal angle of incident light were varied and the optical response of the devices was investigated. At the optimal S-orientation corresponding to $90^{\circ}$ azimuthal angle the tilting resulting in the highest absorptance was determined. 
In these optimal detector orientations the spectral sensitivity for both p-polarized light and the polarization contrast as well as its spectral sensitivity were investigated, since these are crucial SNSPD properties in quantum communication and cryptography applications.

\section{Results}

The absorptance in the original / partially / completely optimized NCAI-SNSPD are 73.7\% / 74.0\% / 74.7\%, which are achieved at the $81.0^{\circ} / 82.1^{\circ} / 81.7^{\circ}$ plasmonic Brewster angle. At this orientation light tunneling promotes the absorptance enhancement originating from the collective resonances on coupled nano-cavities.

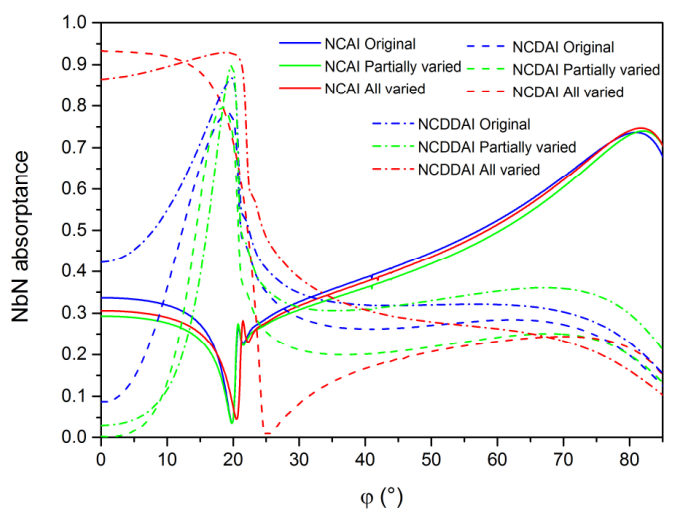

Figure 2. $\mathrm{NbN}$ absorptance as a function of $\varphi$ polar angle in user-defined, in partially ad in completely optimized systems.

The absorptance is modulated at small tilting caused by the appearance of a pseudo plasmonic band-gap, which causes appearance of a global minimum.

In NCDAI-SNSPDs the absorptance modulation is completely reversed, and a plasmonic pass-band appears, where $78.2 \% / 79.7 \%$ and $93.3 \%$ absorptance is achieved at $19.2^{\circ} / 18.4^{\circ} / 0^{\circ}$ tilting.

In all NCDDAI-SNSPD the absorptance is enhanced already at perpendicular incidence, and the maximal absorptance of $86.8 \% / 89.9 \% / 92.9 \%$ is reached at $19.8 \% 19.8 \% 18.9^{\circ}$ tilting.

(a)

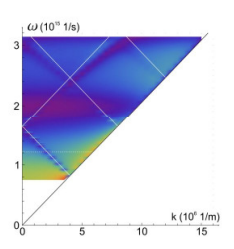

(b)

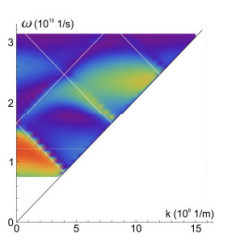

(c)

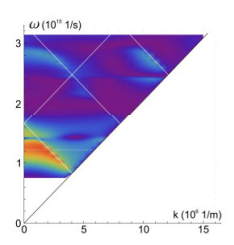

Figure 3. Dispersion relation of NbN absorptance in completely optimized (e) NCAI-, (f) NCDAI- and (g) NCDDAI-SNSPD.

The achieved absorptance is almost polar angle independent at the plasmonic Brewster angle in NCAI-SNSPD (Fig. 3a). The plasmonic pass-bands originates from backward propagating plasmonic waves, which are grating coupled in first order on the deflector arrays (Fig 3b, c).

The achieved polarization contrast is $10^{2} 10^{3} / 10^{5}$ in original, partially and completely optimized NCAI/NCDAI-/NCDDAI-SNSPDs, respectively.

\section{Conclusion}

All integrated plasmonic structures result in considerable absorptance enhancement. The advantage of deflectors is the appearance of large absorptance at small tilting, besides this double deflecotors result in enormous polarization contrast enhancement as well.

\section{References}

[1] X. Hu, E. A. Dauler, R. J. Molnar, K. K. Berggren „Superconducting nanowire singlephoton detectors integrated with optical nano-antennae” Optics Express 19(1) 17-31 (2011)

[2] M. Csete, Á. Sipos, A. Szalai, F. Najafi, G. Szabó. K.K. Berggren, "Improvement of infrared single-photon detectors absorptance by integrated plasmonic structures," Scientific Reports 3:2406 (2012)

[3] J. A. Sánchez-Gil and A. A. Maradudin, "Near-field and far-field scattering of surface plasmon polaritons by one-dimensional surface defects," Phys. Rev. B 60, 8359 (1999). 